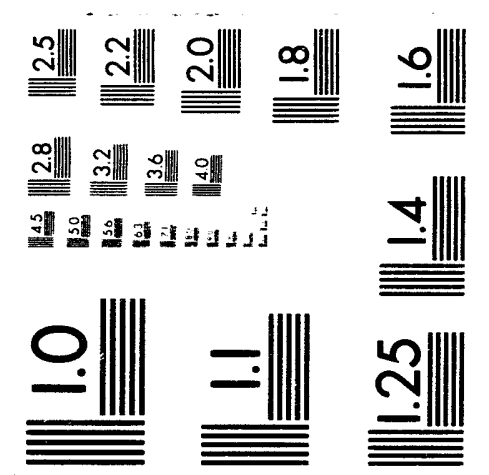



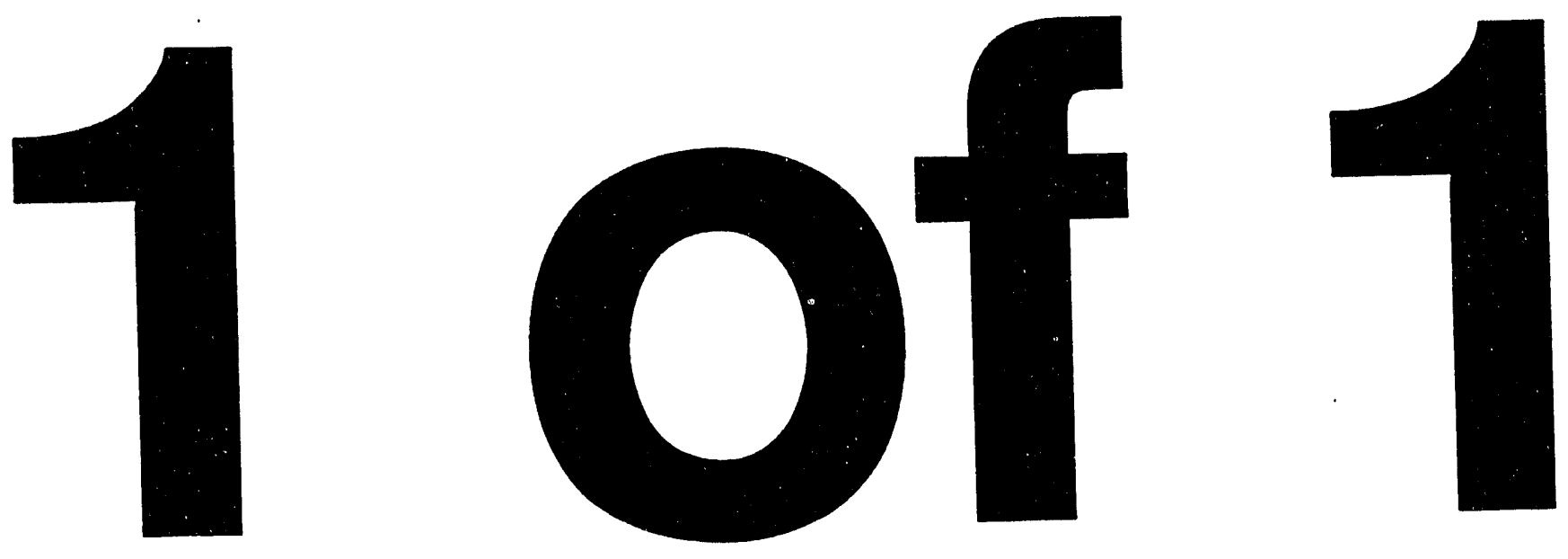
UCRL-ID-113852

\title{
The DEFUZE Code for Modeling Gaseous Diffusion of Volatile Contaminants in the Vadose Zone
}

\author{
February 1994
}

\author{
John J. Nitao
}

\begin{abstract}
DISCLAIMER
This report was prepared as an account of work sponsored by an agency of the United States Government. Neither the United States Government nor any agency thereof, nor any of their employees, makes any warranty, express or implied, or assumes any legal liability or responsibility for the accuracy, completeness, or esefulness of any information, apparatus, prociuct, or process disclosed, or represents that its use would not infringe privately owned rights. Reference herein to any specific commercial product, process, or service by trade name, trademark, manufacturer, or otherwise does not necessarily constitute or imply its endorsement, recommendation, or favoring by the United States Government or any agency thereof. The views and opinions of authors expressed herein do not necessarily state or reflect those of the United States Government or any agency thereof.
\end{abstract}




\section{Contents}

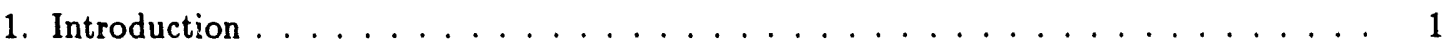

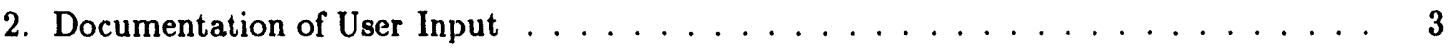

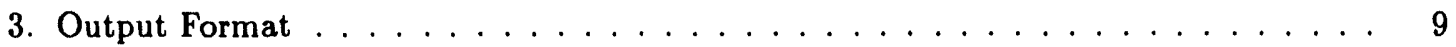

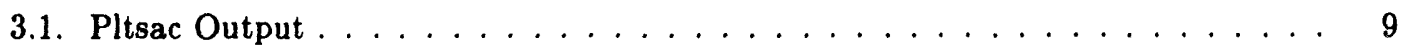

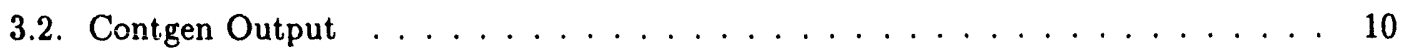

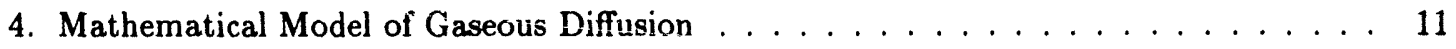

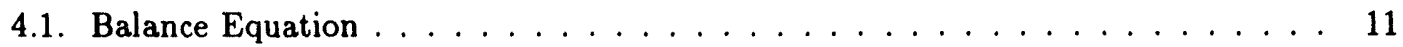

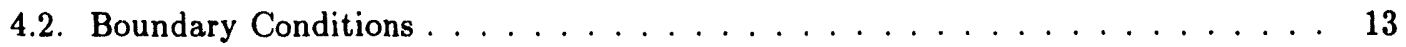

4.3. Partitioning at the Water Table . . . . . . . . . . . . . . . . . 13

4.4. Anisotropy in the Apparent Diffusion Coefficient . . . . . . . . . . . 14

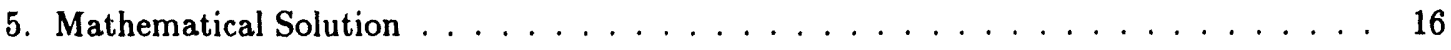

6. Specifying the Initial Contaminant Distribution $\ldots \ldots \ldots \ldots \ldots \ldots$

6.1. Method A: Specifying Contaminant Distribution through First and Second Mom-

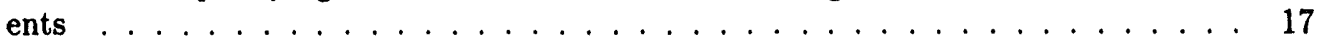

6.2. Method B: Specifying Contaminant Distribution from Borehole Data . . . . . . 18

6.3. Method C: Specifying Contaminant Distribution from Borehole Data . . . . . . . 20

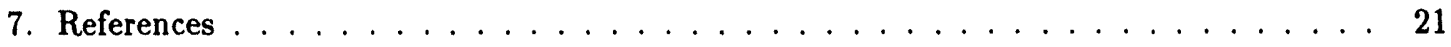

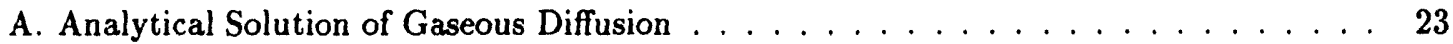

B. Sample Problem $\ldots \ldots \ldots \ldots \ldots \ldots \ldots \ldots \ldots$

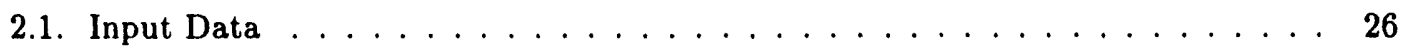

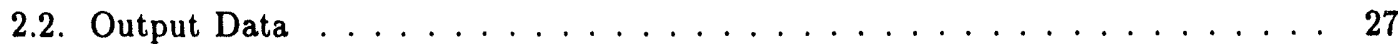




\section{Introduction}

The DEFUZE computer model is a convenient and simple tool for calculating the subsurface gaseous diffusion of volatile contaminants in the vadose zone for the purposes of guiding vadose zone characterization and data collection efforts and for determining the need for site remediation. The reader is referred to Nitao (1990) for further details on the conceptual model for contaminant transport in the vadose zone and an application of DEFUZE to a site at the Lawrence Livermore National Laboratory.

Highly soluble and volatile contaminants, such as trichloroethylene (TCE), may be present in the vadose zone in several forms: as a solute in the aqueous phase, a vapor in the gas phase, an adsorbate on the solid phase, or a nonaqueous liquid phase (free product). In general, contaminant transport can occur by advection and diffusion in all phases that are present. However, at sites where infiltration is small and no free phase is present, gaseous diffusion will be the dominant mode of transport. Infiltration is not important at sites situated in arid and semiarid climates nor at sites with a paved ground surface.

DEFUZE solves the transport of a single volatile soluble component through gaseous diffusion in a homogeneous vadose zone domain. The effective diffusion coefficient can have a constant vertical-horizontal anisotropy. Movement by gaseous and liquid advection is not treated, neither is the presence of a free phase. Given the initial concentration distribution, the model predicts the resulting aqueous concentration at the top of the water table, the contaminant ground surface flux, and the resulting movement of soil concentrations in the vadose zone. Solid sorption of the contaminant is treated by a linear isotherm $\left(K_{d}\right)$. Part: ioning between aqueous and gas phases is treated by use of Henry's constant. The upper boundary of the domain can be treated either as open to the atmosphere (zero concentration) or as a sealed surface (zero flux). The flux through the bottom boundary, which is the water table, is considered sufficiently negligible, compared to fluxes in the vadose zone, so that, with regards to vadose zone transport, it is treated as a no-flow boundary. The concentration at the water table is obtained from partitioning the total soil concentration at the lower boundary of the vadose zone to aqueous concentration. No attenuation across the water table capillary transition zone is included. Conditions under which these approximations are vaiid are discussed in Nitao (1990). If attenuation is desired, the concentrations that are output from the model can be used as input to a saturated zone model, using a mass transfer coefficient approach across the transition zone (Nitao, 1990). 
The analytical solution method represents the contaminant distribution as a sum of gaussianlike solutions of the diffusion equation in a cylindrical coordinate system. The "method of images" is used to satisfy the upper and lower boundary conditions. The initial shape and size of the concentration distribution is a gaussian function in the $r$ and $z$ coordinates and can, therefore, be conveniently specified by the user through a variety of methods, including specification of total mass and second moments of the contaminant distribution. This feature is especially convenient because the exact distribution of contaminants, in practice, is uncertain due to the sparse distribution of the sampling points. With this model, one can easily vary the amount, location, and possible vertical and horizontal size of the contaminant distribution to see the effects on the concentrations at the water table. One can also easily vary other properties such as liquid saturation and soil properties.

The program is written in the $\mathrm{C}$ language and currently runs under Unix. It should be highly portable to other operating systems.

\section{Acknowledgment}

This work was supported by the Environmental Restoration Division at the Lawrence Livermore National Laboratory through the Vadose Zone Investigation Program (VZIP) under the direction of John Ziagos and Dorothy Bishop. The author's work benefited greatly through interactions with his colleagues in the VZIP, especially Joe Iovenitti of Weiss Associates. 


\section{Documentation of User Input}

The program is run by typing the following command:

defuze < input-file > output-file

which reads and writes data using the specified files.

\section{INPUT FILE RULES:}

1. Input is free format except that line breaks have significance. The order in which the data appear also matters.

2. A data line typically begins with a keyword, which starts with a '\$' as its first character. The data must immediately follow on the same line and be separated from the keyword by at least one blank character. For example,

$$
\text { \$porosity } 0.30
$$

sets the data associated with the key word \$porosity to the value 0.30 . Any characters after the data are ignored and can be used for comments. For example,

$$
\text { sporosity } 0.30 \text { Iractional porosity }
$$

In some cases where multiple line data are being read, the data come after the keyword. For example,

$$
\begin{aligned}
& \text { \$data } \\
& \text { 10. 10. } 1000 \text {. } \\
& \text { 20. 10. } 2000 \text {. } \\
& \text { 30. } 10.500 \text {. } \\
& \text { 40. 10. } 400 \text {. }
\end{aligned}
$$

An exception to the above rules is the reading-in of the run title, which has the run title occupying the next line after the keyword. For example,

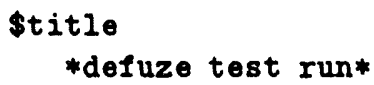

3. Data can begin in any column, but must end before column 200 .

4. Physical units are in the M-K-S system except that, in some cases, time has units of years instead of seconds. Concentrations are in units of parts per billion (mass/mass). 
5. The character $\%$ serves as a "comment" character. That is, all characters after a $\%$ are ignored to the end of the $l$ ae, and lines which begin with a \% are ignored.

6. Blank lines are ignored.

Input can be divided into three sections in the following order:

1. Physical and geometric properties.

2. Options for specifying initial concentration distribution. Possible options are: $\$$ initConc, \$initConcMid, \$Moments.

3. Output options. Possible options are:

\$contour, \$HTpeak, \$WTprofile, \$WTVertProfile, \$SurfFlux, \$SurfFluxDist

A sample input file is given in Appendix B.. The following is a documentation of the input file. Sample numeric data values are used to help denote the type of data that is needed.

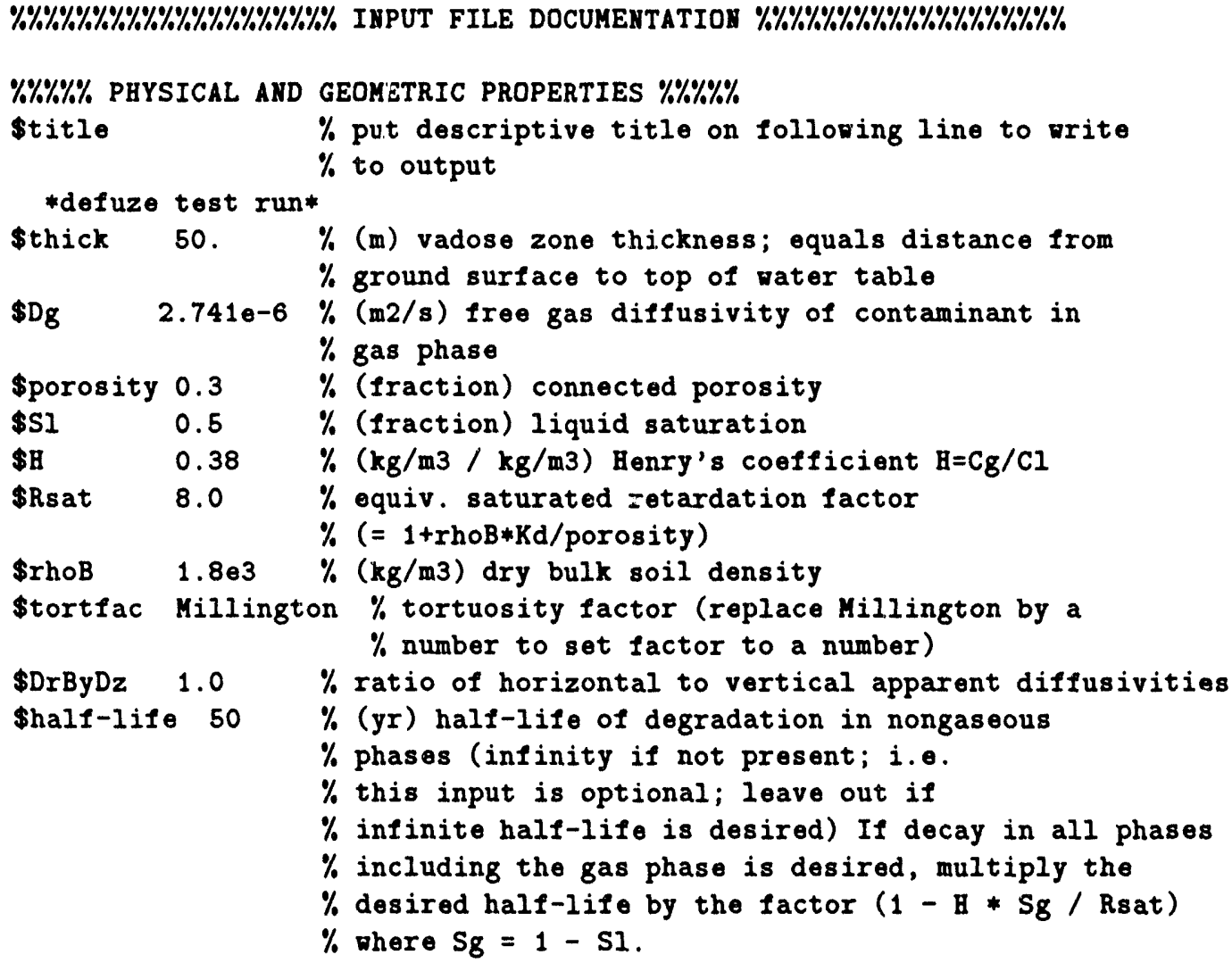




\$surface open $\%$ if "open" then ground surface is open;
\% if "closed" then it is sealed
$\$ 10 g$ if "on" then concentration is alnays output
$\%$ as log 10 of conc.; if "off" no log is taken;
$\%$ does not affect surface flux output

$\% \% \%$ IMITIAL CONC. OPTIONS $\% \% \%$

$\%$

\% MOTE: only one of the following options should be specified.

$\%$

$\%$ init. conc. option: \$Moments

$\%$ Set initial concentrations by specifying first and

$\%$ second moments of the contaminant distribution -- Method $A$.

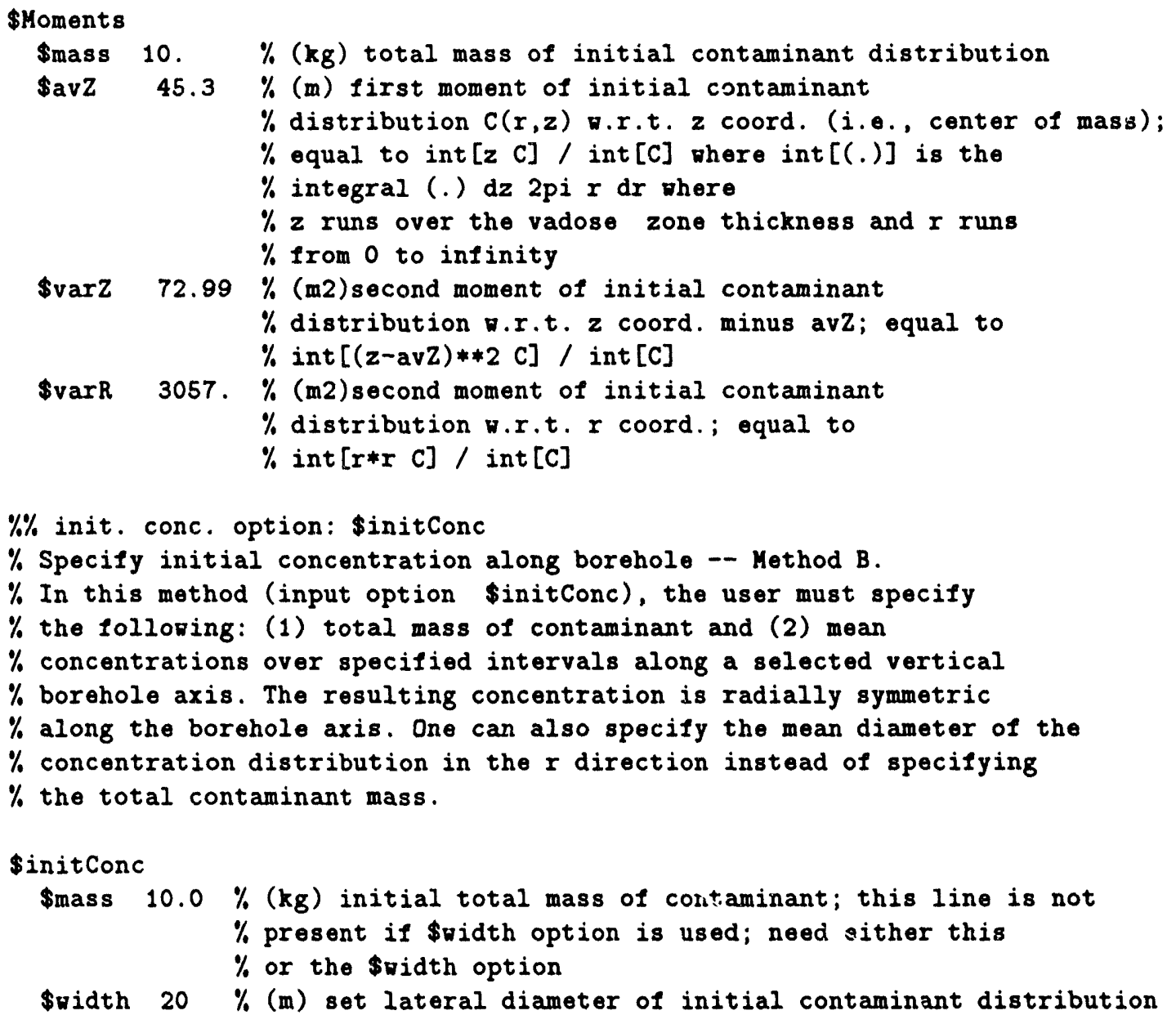




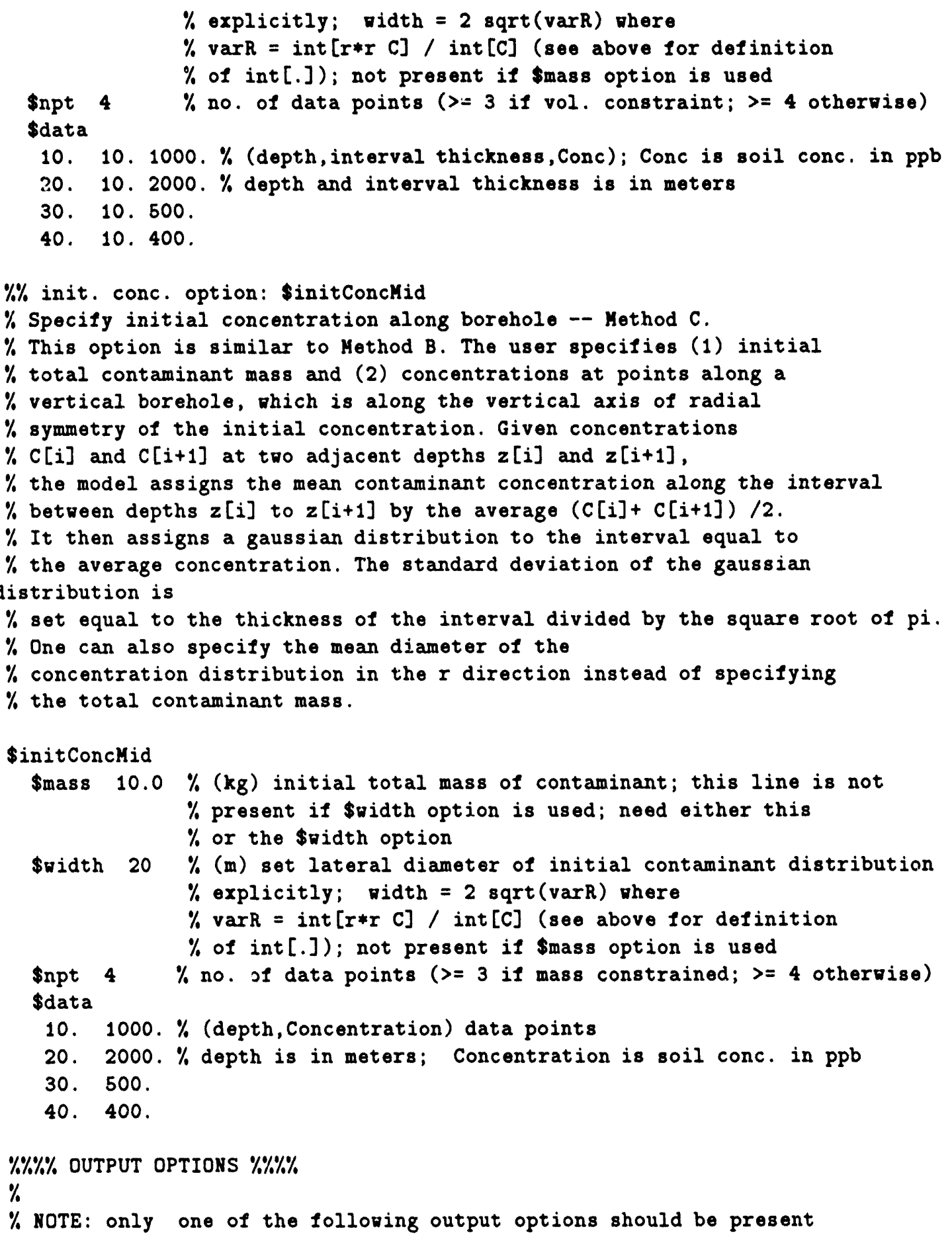


$\%$ output option: SWTpeak

$\%$ The following produces time (first column) versus aqueous concentrations

$\%$ (second column) at the point on the water table which is the

$\%$ intersection of the vater table with the vertical centerline

$\%$ of the initial concentration distribution. The concentration

$\%$ vill be a maximum vith respect to the vater table at this point.

$\%$ (Output is a pltsac output file. This format is explained

$\%$ in a later section.)

\begin{tabular}{|c|c|c|c|}
\hline peas & & & \\
\hline $\begin{array}{l}\text { \$tbnd } \\
\$ d t\end{array}$ & $\begin{array}{l}0.030 \\
5 .\end{array}$ & $\begin{array}{l}\%(y r) \\
\%(y r)\end{array}$ & $\begin{array}{l}\max \text { and min times } \\
\text { time increment }\end{array}$ \\
\hline
\end{tabular}

$\%$ output opision: \$WTprofile

$\%$ The following produces radial distance from the contaminant

$\%$ distribution centerline (first column) versus the aqueous

$\%$ concentration (mass/mass) profile at the vater table as a function

$\%$ of radial distance from the central vertical axis of the spill;

$\%$ this is done for several time periods. (Output is a pltsac

$\%$ output file. This format is explained in a later section.)

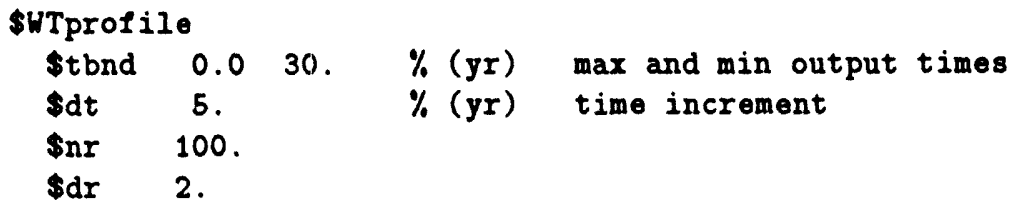

$\%$ output option: \$VertProfile

$\%$ The following produces the vertical depths (first column) of several points

$\%$ along the centerline of the contaminant distribution versus the

$\%$ total soil concentration profile at each point (second column) at

$\%$ various time periods. (Output is a pltsac output file. This format

$\%$ is explained in a later section.)

\$VertProfile

$\begin{array}{lccll}\text { \$tbnd } & 0.0 & 30 & \%(\mathrm{yr}) & \max \text { and min output times } \\ \$ \mathrm{dt} & 5 . & & \%(\mathrm{yr}) & \text { time increment } \\ \$ \mathrm{nz} & 110 & & \% \text { no. of output points on vertical axis }\end{array}$

$\%$ output optior: \$SurfFlux

$\%$ The following produces time (first column) versus

$\%$ the cumulative total surface mass flux (second column)

$\%$ divided by initial mass of the contaminant. 
$\%$ (Output is a pltsac output file. This format is explained

$\%$ in a later section.)

$\begin{array}{lllll}\begin{array}{l}\text { \$SurfFlux } \\ \text { \$tbnd }\end{array} & 0.0 & 30 & \%(\mathrm{yr}) & \max \text { and min times } \\ \text { \$dt } & 5 . & & \%(\mathrm{gr}) & \text { time increment }\end{array}$

$\%$ output option: \$contour

$\%$ The folloving produces the total soil concentrations (mass/mass)

$\%$ at points on a rectangular grid at the specified time.

$\%$ It can be used for contouring the concentrations. (Output is in

$\%$ contgen format. This format is explained in a later section.)

$\%$ The model does not directly use snumcon and \$conlevels. They only are

$\%$ passed to output.

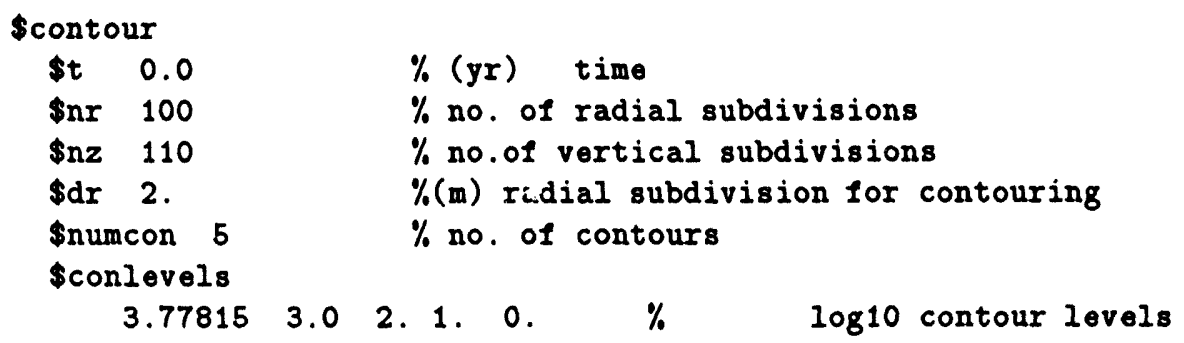

$\%$ output option: \$SurfFluxDist

\% The following produces the radial distance from the center line of the spill

$\%$ versus the contaminant surface mass flux per unit area along the ground

$\%$ surface at specified times. (Output is a pltsac output file. This

$\%$ format is explained in a later section.)

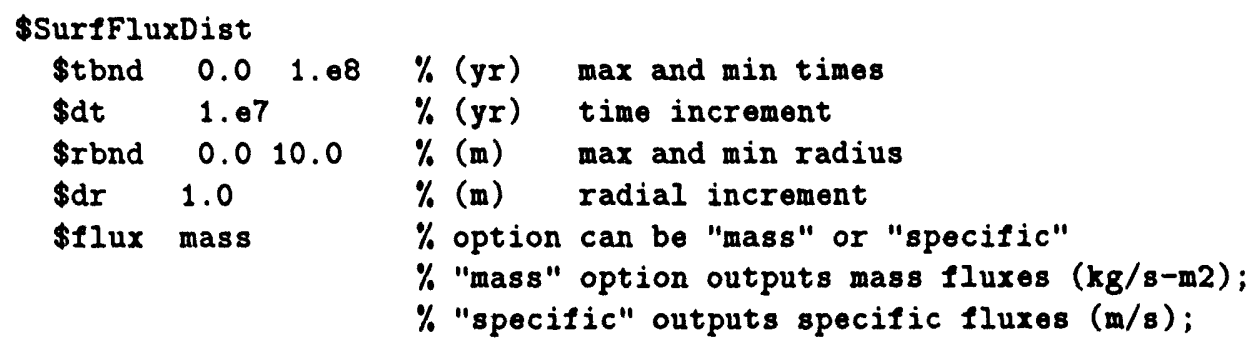

$\% \% \% \% \% \% \% \% \% \% \% \% \% \% \% \% \% \% \%$ END OF INPUT DOCUMENTATION $\% \% \% \% \% \% \% \% \% \% \% \% \% \% \% \% \% \% \% \% \% \% \%$ 


\section{Output Format}

\subsection{PItsac Output}

The pltsac output format is used to represent an $x-y$ set of data pairs suitable for plotting a curve or a set of curves. It is of the following form:

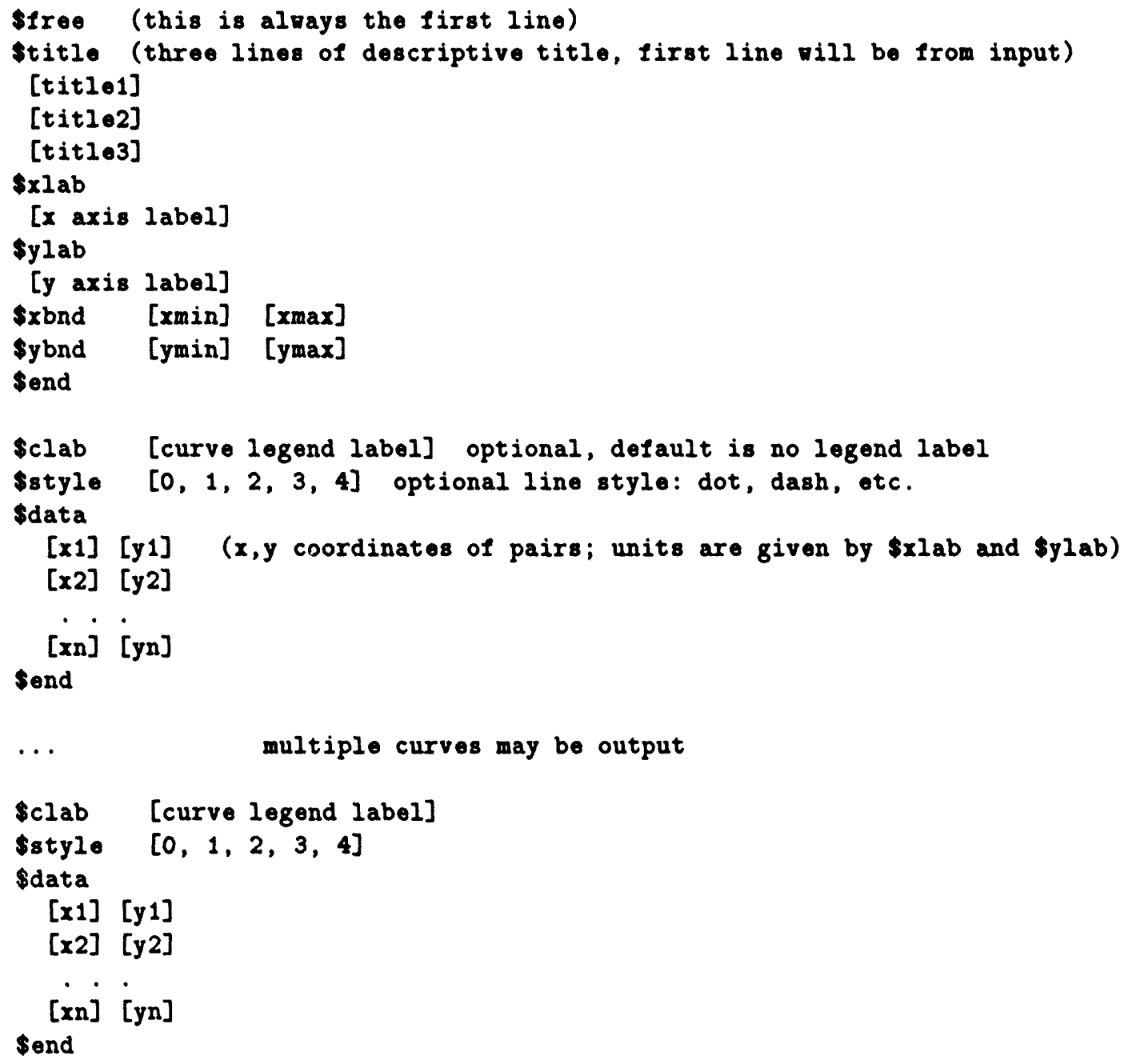




\subsection{Contgen Output}

The contgen output format is for representing data defined over a rectangular grid (we will call it the contour grid for short) that can be used for plotting a contour graph, for example, a concentration field. It is of the form:

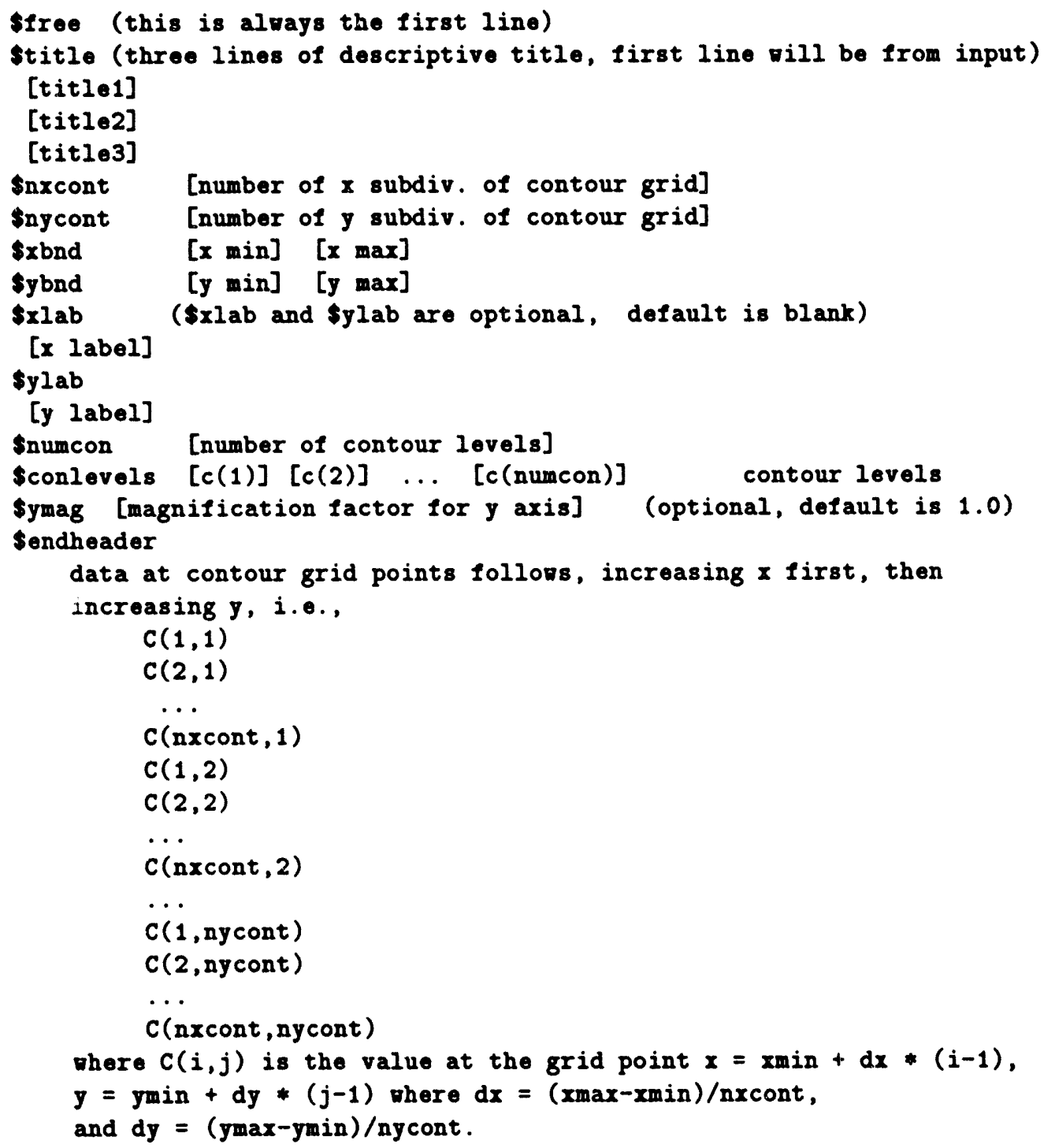




\section{Mathematical Model of Gaseous Diffusion}

In this section, we present the basic partial differential equation and initial boundary value problem solved by the DEFUZE code.

\subsection{Balance Equation}

Consider the balance equation for a single volatile component in a two-fluid phase porous medium. Neglecting gaseous and liquid advection and molecular diffusion in the liquid phase, the balance equation is

$$
\frac{\partial}{\partial t}\left[\phi\left(S_{\ell} C_{\ell}+S_{g} C_{g}\right)+C_{s}\right]=\nabla \cdot \phi S_{g} \tau_{g} D_{g} \rho_{g} \nabla \omega_{g}-\lambda\left[\phi S_{\ell} C_{\ell}+C_{s}\right]
$$

where

$\ell=$ subscript, aqueous phase,

$g$ = subscript, gas phase,

$s$ = subscript, adsorbed solid or solid phase,

$C=$ component concentration in the respective phase (mass/volume),

$\omega=$ mass fraction,

$\rho=$ mass density,

$\phi=$ porosity,

$\tau=$ tortuosity factor,

$D=$ free diffusion coefficient,

$\lambda=$ decay constant for decay assumed to occur only in aqueous and adsorbed phases $(=\ln (2) /($ half-life $))$.

We assume local thermodynamic equilibrium and that Henry's law is valid,

$$
\frac{C_{g}}{C_{\ell}}=H
$$

and assume a linear partitioning between the solid and aqueous phases for adsorbed component on the solid

$$
C_{s}=\rho_{B} K_{d} C_{\ell},
$$

where

$H=$ i'enry's constant,

$\rho_{B}=$ bulk try porous medium density, and

$K_{d}=$ partitioliing coefficient. 
The tortuosity factor $r_{g}$ is a function of saturation and the pore space geometry. The model has an option of setting the tortuosity factor either to a constant number or can be calculated from the Millington (1990) formula

$$
\tau_{g}=\phi^{1 / 3} S_{g}^{7 / 3}
$$

Contaminant concentrations in the vadose zone are usually measured in units of total soil concentration, $\omega_{T}$, equal to mass of contaminant per total mass of soil, i.e.,

$$
\omega_{T}=\frac{\phi\left(S_{\ell} C_{\ell}+S_{g} C_{g}\right)+C_{g}}{\phi\left(\rho_{\ell} S_{\ell}+\rho_{g} S_{g}\right)+\rho_{B}} .
$$

Assuming homogeneous soil properties and uniform water saturation, Eq. (4.1) can be written in terms of the total soil concentration as

$$
\frac{\partial \omega_{T}}{\partial t}=D_{a p p} \nabla^{2} \omega_{T}-\lambda_{a p p} \omega_{T}
$$

where the "apparent diffusivity" is given as

$$
D_{a p p}=\frac{S_{g} \tau_{g} D_{g} H}{R}
$$

and the "apparent decay constant" as

$$
\lambda_{\text {app }}=\lambda\left(1-H S_{g} / R\right) .
$$

The unsaturated retardation factor in these equations is defined by

$$
R=S_{\ell}+H S_{g}+\rho_{B} K_{a} / \phi
$$

The apparent decay constant is different from the decay constant $\lambda$ because we allow decay to occur only in the solid and aqueous phases and not in the gaseous phase. They would be equal if decay occurred in both phases (at equal rates).

The decay constant is related to the half-life $T$ through the relationship

$$
\lambda=\ln (2) / T \text {. }
$$

The user inputs the value of $T$ corresponding to decay in the aqueous and solid phases. No decay is assumed to be present in the gas phase. No decay is present in the gas phase. If decay in the gas phase is desired as well, one should input the decay half-life $T$ multiplied by the factor $\left(1-H S_{g} / R\right)$.

To see this, note that the apparent degradation half-life of the contaminant, $T_{a p p}$, is given by

$$
T_{a p p}=\frac{\ln (2)}{\lambda_{a p p}} .
$$


Note that

$$
\frac{T_{\text {app }}}{T}=\frac{\lambda}{\lambda_{\text {app }}}
$$

where $T$ is the half-life in the aqueous and solid phases, so that the apparent decay half-life is related to the decay half-life $T$ by

$$
T_{\text {app }}=T /\left(1-H S_{g} / R\right) .
$$

\subsection{Boundary Conditions}

The diffusion equation presented in the previous subsection is solved within a domain in cylindrical $(r, z)$ coordinates defined over a horizontal infinite strip with upper boundary either open or closed to the atmosphere. The upper boundary is set at $z=0$ and the lower boundary at $z=L$ with $L$ equal to the vadose zone thickness.

Neglecting advective flow, the open atmosphere at the upper boundary, is treated as a zero concentration boundary

$$
\omega_{T}(z=0)=0 \text {. }
$$

This condition neglects the stagnant boundary layer, which acts as a buffer zone between the ground surface and the well-mixed atmosphere with essentially zero concentration. The contaminant will diffuse from the ground surface and through this boundary layer. This diffusion resistance is much less than that of an equivalent thickness of soil. The tortuosity factor of dry porous media are typically on the order of 0.1 to 0.001 , while the boundary layer effectively has a tortuosity factor of 1.0. Thus, the latter becomes significant only when the distance over which subsurface diffusion from the high concentration regions to the ground surface is less than 0.1 to 0.001 times the thickness of the boundary layer. (Wet soil will have even smaller tortuosity factors.) The boundary layer is typically on the order of, at most, a few feet. Therefore, Eq. (4.14) is a good assumption except when there are high concentrations only a few inches from the soil surface.

The boundary condition for a closed surface at the ground surface is given by

$$
\frac{\partial \omega}{\partial z}(z=0)=0 \text {. }
$$

This condition is appropriate for a paved surface where diffusion, if any, occurs at a much reduced rate. A completely sealed boundary condition is conservative with respect to predicting higher concentrations at the water table.

\subsection{Partitioning at the Water Table}

The model assumes that the soil concentration gradient across the capillary transition zone lying between the vadose zone and the saturated zone is neglible compared to gradients in the saturated zone just below the water table. The reader is directed to Nitao and Iovenitti (1990) 
for conditions under which this assumption holds. The assumption is valid as long as the vertical dispersive fluxes in the saturated zone are small compared to the diffusive fluxes in the transition zone. This holds exactly if there is no ground water flow and hence no hydrodynamic dispersion; only liquid molecular diffusion is the transport mechanism, which is small compared to gaseous diffusion. If the assumption does not hold, the model will predict higher concentrations at the top of the saturated zone than actual.

Since contaminant concentrations are usually expressed in terms of aqueous concentration, i.e., mass of contaminant per mass of aqueous solution, we convert from soil concentration of contaminant at the water table to aqueous concentration, $\omega_{T}$, from the formula

$$
\omega_{\ell}=\left(\frac{\rho_{s}}{\phi R_{s a t}}\right) \omega_{T},
$$

where the wet bulk soil density is given by

$$
\rho_{s}=\phi\left(\rho_{\ell} S_{\ell}+\rho_{g} S_{g}\right)+\rho_{B} .
$$

To a good approximation, we may take $\rho_{\ell}$ to be the density of pure water, and we may neglect the gas phase term in the expression for $\rho_{\mathrm{a}}$. The saturated retardation factor is defined as

$$
R_{\text {sat }}=1+\rho_{B} K_{d} / \phi \text {. }
$$

\subsection{Anisotropy in the Apparent Diffusion Coefficient}

Treatment of heterogeneity in the soil properties is restricted in the model to anisotropy in the apparent diffusivity uniform in space. We show how to set the anisotropy ratio for an idealized vadose zone with two alternating horizontal layers of soils with thickness $\ell_{1}$ and $\ell_{2}$ and soil properties

$$
\left(S_{g}\right)_{i}, \phi_{i},\left(\tau_{g}\right)_{i},\left(\rho_{B} K_{d} / \phi\right)_{i} \quad i=1,2 .
$$

Define the relative thicknesses of the two soil types as

$$
\gamma_{1}=\ell_{1} /\left(\ell_{1}+\ell_{2}\right)
$$

and

$$
\gamma_{2}=\ell_{2} /\left(\ell_{1}+\ell_{2}\right)
$$

We assume that the layer thicknesses are sufficiently small compared to the scale of interest. The apparent diffusivity can then be represented as a composite material with the "parallel resistor" representation for the horizontal diffusivity

$$
D_{h}=\left[\left(\gamma \phi S_{g} \tau_{g}\right)_{1}+\left(\gamma \phi S_{g} \tau_{g}\right)_{2}\right] \frac{H}{R_{c} \phi_{c}} D_{g}
$$

and the vertical diffusivity given by the "series resistor" representation,

$$
D_{v}=\left[\frac{1}{\left(\frac{1}{\gamma \phi S_{g} \tau_{g}}\right)_{1}+\left(\frac{1}{\gamma \phi S_{g} r_{g}}\right)_{2}}\right] \frac{H}{R_{c} \phi_{c}} D_{g} .
$$


The composite properties are defined as

$$
\phi_{c}=(\gamma \phi)_{1}+(\gamma \phi)_{2}
$$

and

$$
R_{c}=(\gamma R)_{1}+(\gamma R)_{2},
$$

where

$$
R_{i}=\left(S_{\ell}\right)_{i}+H\left(S_{g}\right)_{i}+\left(\rho_{B} K_{d} / \phi\right)_{i} \quad i=1,2 .
$$

The resulting anisotropy ratio required as input to the model is given by

$$
\frac{D_{h}}{D_{v}}=\left[\left(\gamma \phi S_{g} \tau_{g}\right)_{1}+\left(\gamma \phi S_{g} \tau_{g}\right)_{2}\right]\left[\left(1 / \gamma \phi S_{g} \tau_{g}\right)_{1}+\left(1 / \gamma \phi S_{g} \tau_{g}\right)_{2}\right]
$$




\section{Mathematical Solution}

A detailed description of the solution to the gaseous diffusion equation [Eq. (4.6)] is given in Appendix A.. DEFUZE represents the solution as a sum of gaussian-like functions, including the addition of "image" solutions in accordance with the well-known technique called "the method of images" for satisfying the boundary conditions.

In cylindrical coordinates $(r, z)$, let $z=0$ be the ground surface and $z=L$ be the water table where $L$ is the vadose zone thickness. The solution for an open upper boundary is of the form

$$
\omega(r, z, t)=\sum_{k=1}^{N} \sum_{n=-\infty}^{\infty}\left[(-1)^{n} F_{k}\left(r, z-2 n L-a_{k}, t\right)+(-1)^{n+1} F_{k}\left(r, z-2 n L+a_{k}, t\right)\right],
$$

where $F_{k}(r, z, t)$ are solutions to the diffusion equation of the form,

$$
F_{k}(r, z, t)=b_{k} R(r, t) Z_{k}(z, t)
$$

with

$$
Z_{k}(z, t)=\frac{\exp \left[-\left(z-a_{k}\right)^{2} / 4\left(t D_{a p p}+h_{k}^{2} / 2\right)\right]}{\sqrt{t D_{a p p}+h_{k}^{2} / 2}}
$$

and

$$
R(r, t)=\frac{\exp \left[-r^{2} / 4\left(t D_{a p p}+w^{2} / 2\right)\right]}{t D_{a p p}+w^{2} / 2}
$$

For a closed upper boundary, we have

$$
\omega(r, z, t)=\sum_{k=1}^{N} \sum_{n=-\infty}^{\infty}\left[F_{k}\left(r, z-2 n L-a_{k}, t\right)+F_{k}\left(r, z-2 n L+a_{k}, t\right)\right] .
$$

In the code, the infinite sum is truncated when the contribution of the terms becomes small relative to the cumulative sum.

The contants $b_{k}, a_{k}$, and $h_{k}$ are selected so that the solution at $t=0$ is the desired initial concentration distribution or is a suitable approximation. 


\section{Specifying the Initial Contaminant Distribution}

The solution is a sum of solutions given by either Eq. (5.28) or Eq. (5.32). Although the initial concentration distribution is gaussian-like, the initial shape is complicated by the effect of the boundary conditions. That is, the concentration distribution is not simply a sum of gaussians centered within the problem domain, but also includes contributions from gaussians centered outside the problem domain from image solutions. The code must therefore provide means for specifying the initial contaminant distribution through the parameters $w, h_{k}$, and $a_{k}$ in Eqs. (5.28) and (5.32). We now describe three separate methods available in the DEFUZE code.

\subsection{Method A: Specifying Contaminant Distribution through First and Second Moments}

In this method (input option \$Moments), the user specifies the following quantities: (I) initial total mass of contaminant, (II) the mean $E_{z}[\omega]$ of the initial contaminant concentration, $\omega(r, z, 0)$, in the $z$ direction,

$$
E_{z}[\omega]=\frac{\int_{0}^{L} \int_{0}^{\infty} z \omega(r, z, 0) 2 \pi r d r d z}{\int_{0}^{1} \int_{0}^{\infty} \omega(r, z, 0) 2 \pi r d r d z},
$$

(III) the spatial variance $\operatorname{Var}_{2}[\omega]$ given by

$$
\operatorname{Var}_{z}[\omega]=\frac{\int_{0}^{L} \int_{0}^{\infty}(z-a)^{2} \omega(r, z, 0) 2 \pi r d r d z}{\int_{0}^{L} \int_{0}^{\infty} \omega(r, z, 0) 2 \pi r d r d z}
$$

and (IV) the variance $\operatorname{Var}_{r}[\omega]$ given by

$$
\operatorname{Var}_{r}[\omega]=\frac{\int_{0}^{L} \int_{0}^{\infty} r^{2} \omega(r, z, 0) 2 \pi r d r d z}{\int_{0}^{1} \int_{0}^{\infty} \omega(r, z, 0) 2 \pi r d r d z} .
$$

Here $L$ denctes the vadose zone thickness.

The above integrals can be approximated numerically. However, in practice there are usually insufficient data to determine them accurately, and it, therefore, makes sense to perform sensitivity studies on these parameters. In this way, the model can be used to determine the value of further contaminant characterization at the site.

This method represents the initial contaminant distribution by a single gaussian centered in the problem domain plus a series of gaussians chosen to satisfy the boundary conditions. That is, we take solution, either Eq. (5.28) or Eq. (5.32), with the $N=1$ and

$$
F_{k}(r, z, t)=b R(r, t) Z(z, t),
$$

where $R(r, t)$ and $Z(z, t)$ are given by Eqs. (5.31) and (5.30), respectively. The resulting contaminant distribution is radially symmetric and centered along the vertical radial axis $r=0$ 
of a cylindrical coordinate system. The ground surface is at $z=0$, and the top of capillary transition zone above water table is at $z=L(z=1$ in dimensionless coordinates).

Because of the boundary conditions, image solutions must be added. Eq. (5.28) is, therefore, a sum of gaussians and not a single gaussian. The mean in the $z$-direction, $E_{z}$, is not equal to $a$, whereas $h$ and $w$ are not directly related to the variances as would be the case for a single gaussian. However, it is possible to perform a numerical iteration process to solve for these parameters such that the means and variances equal the values specified by the user within some tolerance. Essentially, this amounts to finding the root of a system of equations. DEFUZE uses the Newton-Raphson algorithm to perform the iterative numerical solution.

When DEFUZE is running, it will print to the terminal the value of the parameters $a, h$, and $b$ at each iteration. The user should monitor these values to see that they are converging and that the converged values fall within valid ranges, $0<a<L, 0<h<L$, and $b>0$.

\subsection{Method B: Specifying Contaminant Distribution from Borehole Data}

In this method (input option \$initConc), the user specifies the following: (I) total mass of contaminant and (II) mean concentrations over specified intervals along a selected vertical borehole. The resulting concentration is radially symmetric along the borehole axis. One can also specify the variance of the concentration distribution in the $r$ direction instead of specifying the total contaminant mass.

Let there be $N I$ borehole intervals over which the concentrations are specified. Let $\bar{w}_{i}$ be the mean initial concentration of the $i$-th interval, $z_{i}^{\text {mid }}$ the depths of the midpoint of the intervals, and $\Delta z_{i}$ the thicknesses of the intervals. In this method, the solution is first built up of a sum of gaussians, one gaussian for each interval. Image solutions are added so that the solution satisfies the boundary conditions. The vertical width of each gaussian in the $z$ direction is selected as

$$
h_{i}=\Delta z_{i} / \sqrt{2 \pi}
$$

so that there will not be a significantly vertical overlap between the gaussian with the gaussians of other intervals.

In order to determine the coefficients $b_{i}$, we first note that the solution can be written in the form

$$
\omega(r, z, t)=R(r, t) \sum_{i=0}^{N I} b_{i} G_{i}(z, t),
$$

where $G_{i}(z, t)$ is the sum of the gaussian for the $i$-th interval and its image solutions. We then have the approximate relationship resulting from integration with respect to $z$ over the $i$-th borehole interval,

$$
\bar{\omega}_{i} \Delta z_{i}=\int_{z_{i}^{m} z^{m i d}-\Delta z_{i} / 2}^{z_{i}^{m}+\Delta z_{i} / 2} \omega(0, z, 0) d z \approx R(0,0) b_{i} \int_{0}^{L} G_{i}(z, 0) d z .
$$


Since

$$
R(0,0)=2 / w^{2},
$$

we have

$$
b_{i}=\frac{\bar{\omega}_{i} \Delta z_{i}\left(\frac{w^{2}}{2}\right)}{\int_{0}^{L} G_{i}(z, 0) d z} .
$$

The integral in the denominator is calculated numerically by the model.

The above procedure guarantees that the total initial mass along the borehole is approximately equal to the mass computed from the average concentrations in the input, that is,

$$
\int_{0}^{L} \omega(0, z, 0) d z \approx \sum_{i} \bar{\omega}_{i} \Delta z_{i} .
$$

To determine $w$, we note that the total mass of contaminant is given by

$$
\begin{aligned}
M_{0} & =\rho_{s} \int_{0}^{L} \int_{0}^{\infty} \omega(r, z, 0) 2 \pi r d r d z \\
& \approx \rho_{s} \sum_{i} b_{i} \int_{0}^{L} G_{i}(z, 0) d z \int_{0}^{\infty} R(r, 0) 2 \pi r d r d z \\
& \approx \rho_{s} \sum_{i} \bar{\omega}_{i} \Delta z_{i}\left(w^{2} / 2\right)(4 \pi), \text { and } \\
& \approx 2 \pi \rho_{s} w^{2} \sum_{i} \bar{\omega}_{i} \Delta z_{i},
\end{aligned}
$$

from which we get

$$
w=\sqrt{\frac{M_{0}}{2 \pi \rho_{B} \sum_{i} \bar{\omega}_{i} \Delta z_{i}}} .
$$

Here, we used Eq. (6.39) and the fact that

$$
\int_{0}^{\infty} R(r, 0) 2 \pi r d r d z=4 \pi .
$$

Instead of specifying the initial mass of contaminant, the mean lateral diameter $D$ of the contaminant distribution can be set through the formula

$$
D=2 \sqrt{\operatorname{Var}_{r}[\omega]},
$$

where

$$
\operatorname{Var}_{r}[\omega]=\frac{\int_{0}^{L} \int_{0}^{\infty} r^{2} \omega(r, z, 0) 2 \pi r d r d z}{\int_{0}^{L} \int_{0}^{\infty} \omega(r, z, 0) 2 \pi r d r d z}
$$


Substituting Eq. (6.38)

$$
\operatorname{Var}_{r}[\omega]=\frac{\int_{0}^{\infty} r^{2} R(r, 0) 2 \pi r d r}{\int_{0}^{\infty} R(r, 0) 2 \pi r d r}=\frac{w^{2}}{2}
$$

Hence, in the model, we set the parameter $w$ in terms of the diameter $D$ by

$$
w=\frac{D}{\sqrt{2}}
$$

\subsection{Method C: Specifying Contaminant Distribution from Borehole Data}

This option (called input option sinitConcMid) is similar to Method B. The user specifies (1) initial total contaminant mass and (2) concentrations $\omega_{i}$ at points with depth $z_{i}$ along a vertical borehole, which is along the vertical axis of radial symmetry of the initial concentration. In the notation of the previous subsection, the model first sets

$$
\begin{gathered}
\bar{\omega}=\left(\omega_{i+1}+\omega_{i}\right) / 2, \\
z_{i}^{\text {mid }}=\left(z_{i+1}+z_{i}\right) / 2, \quad \text { and } \\
\Delta z_{i}=z_{i+1}-z_{i},
\end{gathered}
$$

and then proceeds as in Method B. 


\section{References}

Millington, R. J. (1990), "Gas Diffusion in Porous Media," Science, 130, 100-102.

Nitao, J. J. and J. Iovenitti (1990), "Modeling of Vadose Zone Migration to Ground Water in the Building 518 and 511 Areas, Appendix G," CERCLA Feasibility Study for the LLNL Livermore Site, W. F. Isherwood, C. H. Hall, M. D. Dresen, eds. (UCRL-AR-104040). 


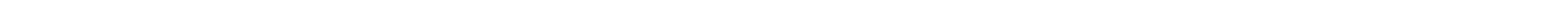


Appendix A

Analytical Solution of Gaseous Diffusion 


\section{A. Analytical Solution of Gaseous Diffusion}

We now present the analytic solution technique used in the model to solve Eq. (4.6). We consider the case where there is no degradation, i.e., $\lambda_{\text {app }}=0$. The solution with degradation included is obtained by multiplying the no-degradation solution by the following factor,

$$
2^{\left(-t / T_{c p}\right)} \text {, }
$$

where $T_{\text {app }}$ is the apparent degradation half-life of the contaminant given by

$$
T_{\text {app }}=\frac{\ln (2)}{\lambda_{\text {app }}} .
$$

Let $z=L$ represent the bottom of the model domain. For convenience, define the dimensionless space and time variables $\left(r^{\prime}, z^{\prime}, t^{\prime}\right)$ by the following substitutions

$$
r^{\prime}=r / L, \quad z^{\prime}=z / L, \quad t^{\prime}=t D_{a p p} / L^{2} .
$$

Note that under this substitution $z$ runs from 0 to 1 . Then Eq. (4.6) becomes

$$
\frac{\partial \omega}{\partial t^{\prime}}=\nabla^{\prime 2} \omega
$$

where we drop the subscript $T$ in $\omega$.

For the remainder of this appendix, we will only deal with dimensionless variables unless explicity stated otherwise, and we drop the primes.

We look for solutions of the form $\omega(r, z, t)=R(r, t) Z(z, t)$. Substituting, we obtain equations for $R(r, t)$ and $Z(z, t)$,

$$
\frac{\partial R}{\partial t}=\frac{1}{r} \frac{\partial R}{\partial r}+\frac{\partial^{2} R}{\partial r^{2}}
$$

and

$$
\frac{\partial Z}{\partial t}=\frac{\partial^{2} Z}{\partial z^{2}}
$$

A family of solutions to these equations that result in a gaussian-like distribution at $t=0$ are given by

$$
Z(z, t)=\frac{\exp \left[-(z-a)^{2} / 4\left(t+h^{2} / 2\right)\right]}{\sqrt{t+h^{2} / 2}}
$$

and

$$
R(r, t)=\frac{\exp \left[-r^{2} / 4\left(t+w^{2} / 2\right)\right]}{t+w^{2} / 2} .
$$

Note that the parameters $a, h$, and $w$ are dimensionless lengths.

The "center of mass" of this distribution is at $r=0, z=a$. The parameter $h$ may be interpreted as the square root of the variance of the distribution at $t=0$ in the $z$ direction,

$$
V a r_{2}[\omega]=h^{2} \text {, }
$$


where we define

$$
\operatorname{Var}_{z}[\omega]=\frac{\int_{0}^{1} \int_{0}^{\infty}(z-a)^{2} \omega(r, z, 0) 2 \pi r d r d z}{\int_{0}^{1} \int_{0}^{\infty} \omega(r, z, 0) 2 \pi r d r d z}
$$

and $w$ is related to the variance in the $r$ direction by

$$
\operatorname{Var}_{r}[\omega]=2 w^{2},
$$

where

$$
\operatorname{Var}_{r}[\omega]=\frac{\int_{0}^{1} \int_{0}^{\infty} r^{2} \omega(r, z, 0) 2 \pi r d r d z}{\int_{0}^{1} \int_{0}^{\infty} \omega(r, z, 0) 2 \pi r d r d z}
$$

By linear superposition, one can take a linear combination of the solutions of the above type to obtain distributions at $t=0$ that are sums of gaussian distributions. To satisfy the boundary conditions at $z=0$ and $z=1$, we also must add "image" solutions in accordance with the well-known technique called "the method of images." The solution for an open upper boundary is of the form

$$
\left.\omega(r, z, t)=\sum_{k=1}^{N} \sum_{n=-\infty}^{\infty}\left[(-1)^{n} F_{k}\left(r, z-2 n-a_{k}, t\right)+(-1)^{n+1} F_{k}\left(r, z-2 n+a_{k}\right), t\right)\right],
$$

where $F_{k}(r, z, t)$ are solutions of the form

$$
F_{k}(r, z, t)=b_{k} R(r, t) Z_{k}(z, t)
$$

with

$$
Z_{k}(z, t)=\frac{\exp \left[-\left(z-a_{k}\right)^{2} / 4\left(t+h_{k}^{2} / 2\right)\right]}{\sqrt{t+h_{k}^{2} / 2}}
$$

and

$$
R(r, t)=\frac{\exp \left[-r^{2} / 4\left(t+w^{2} / 2\right)\right]}{t+w^{2} / 2} .
$$

For a closed upper boundary, we have

$$
\left.\left.\omega(r, z, t)=\sum_{k=1}^{N} \sum_{n=-\infty}^{\infty}\left[F_{k}\left(r, z-2 n-a_{k}\right), t\right)+F_{k}\left(r, z-2 n+a_{k}\right), t\right)\right] .
$$

We can rewrite the solutions in in nondimensionless coordinates. For an open ground surface, the solution is

$$
\omega(r, z, t)=\sum_{k=1}^{N} \sum_{n=-\infty}^{\infty}\left[(-1)^{n} F_{k}\left(r, z-2 n L-a_{k}, t\right)+(-1)^{n+1} F_{k}\left(r, z-2 n L+a_{k}, t\right)\right],
$$

where $F_{k}(r, z, t)$ is of the form

$$
F_{k}(r, z, t)=b_{k} R(r, t) Z_{k}(z, t)
$$


with

$$
Z_{k}(z, t)=\frac{\exp \left[-\left(z-a_{k}\right)^{2} / 4\left(t D_{a p p}+h_{k}^{2} / 2\right)\right]}{\sqrt{t D_{a p p}+h_{k}^{2} / 2}}
$$

and

$$
R(r, t)=\frac{\exp \left[-r^{2} / 4\left(t D_{a p p}+w^{2} / 2\right)\right]}{t D_{a p p}+w^{2} / 2}
$$

For a closed upper boundary, we have, in terms of nondimensionless variables,

$$
\left.\left.\omega(r, z, t)=\sum_{k=1}^{N} \sum_{n=-\infty}^{\infty}\left[F_{k}\left(r, z-2 n L-a_{k}\right), t\right)+F_{k}\left(r, z-2 n L+a_{k}\right), t\right)\right] .
$$

In these equations, the parameters $a_{k}, h_{k}$, and $w$ are nondimensionless and now have units of length. 


\section{B. Sample Problem}

This problem models a vadose zone that is $\mathbf{5 0} \mathrm{m}$ thick. The surface is open to the atmosphere. An isotropic diffusion coefficient is assumed. The contaminant in question is TCE. The total mass of TCE is $10 \mathrm{~kg}$. The initial TCE soil concentrations are specified along a borehole and are set to $1000,2000,0.0,0.0,500$, and $400 \mathrm{ppb}$ at depths of $10,15,15.1,29.9,30$, and $40 \mathrm{~m}$, respectively. Internally, the model will place a gaussian concentration distribution centered at the midpoints between these depths at $12.5,15.05,22.5,29.95$, and $35 \mathrm{~m}$. The average concentrations of these gaussians will be set to be $1500,1000,0.0,250$, and $450 \mathrm{ppb}$, respectively. Note that there are no other gaussians outside of the specified intervals (save for image gaussians centered outside the problem domain, which are added to satisfy the boundary conditions).

Instead of specifying the total mass, it may be desirable to specify the root mean square diameter of the contaminant distribution. If this value were equal to $15 \mathrm{~m}$, one would replace the line

\$mass $10.0 \%$ (kg) total mass of contaminant

by

\$width $15 \%$ (m) mean diameter of contaminant distribution

The output option is set to give the aqueous concentration at the water table at increments of every $5 \mathrm{yr}$ from 0 to $30 \mathrm{yr}$.

\subsection{Input Data}

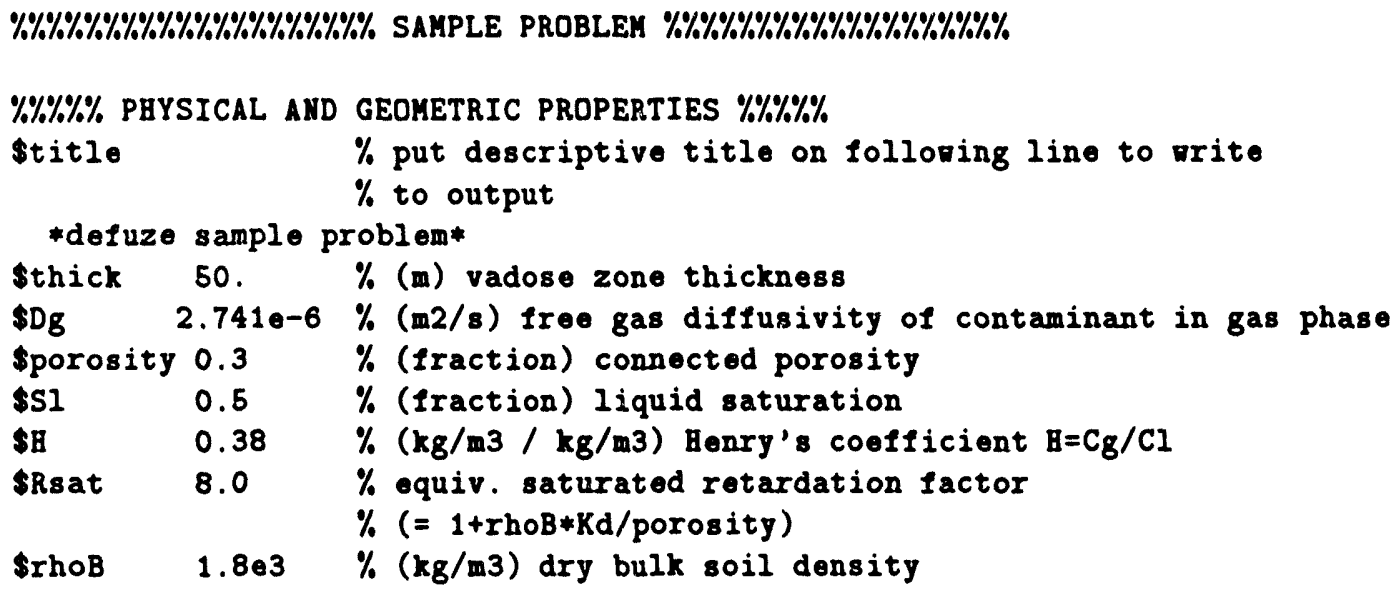




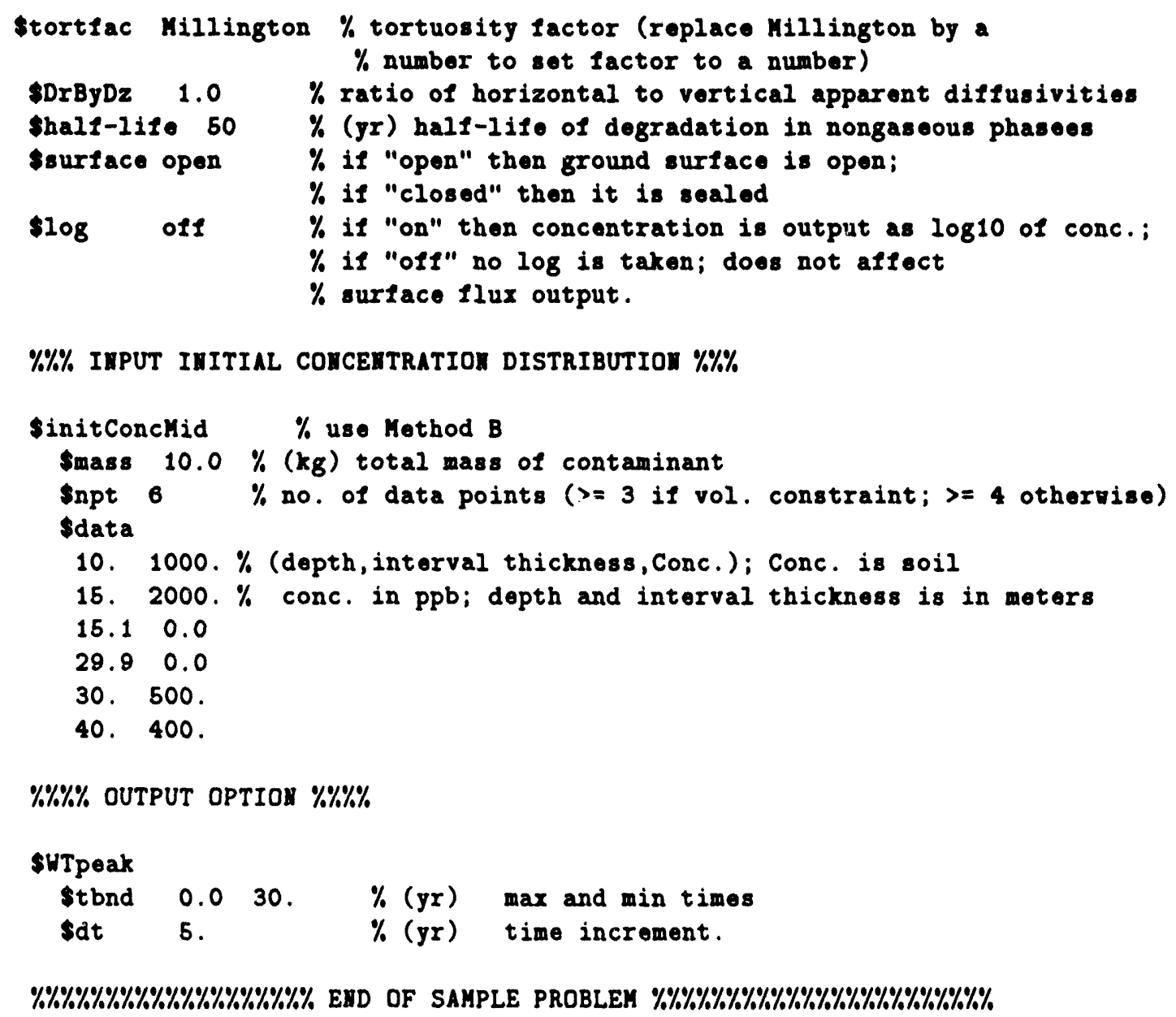

\subsection{Output Data}

The model will output to the terminal (stderr) the following line:

calculated lateral width: $16.408185 \mathrm{~m}$

which gives the computed root-mean square width of the initial contaminant distribution. If the option to input the width using the \$ridth option is used instead, the model will output the computed total mass of the initial distribution. 
The contents of the directed output file are:

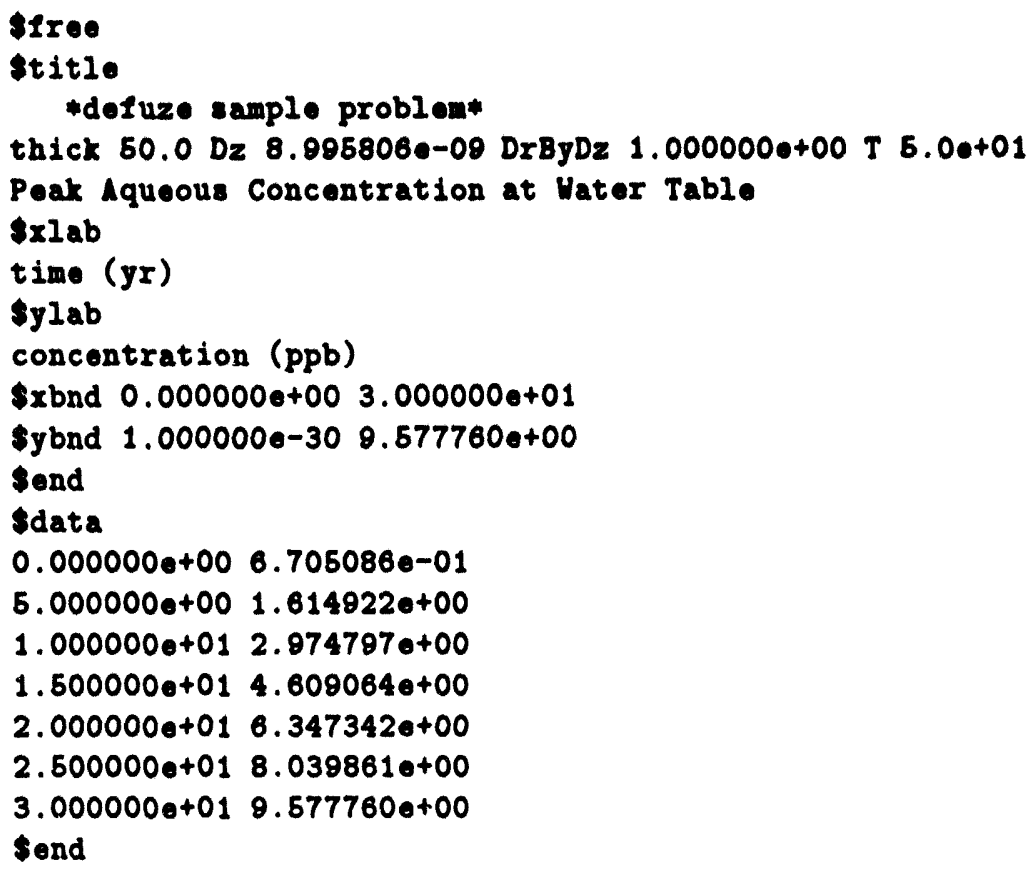

The parameters in the title header are

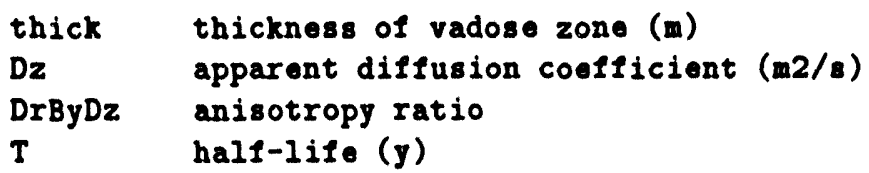


